\title{
Performance of Osteoporosis Self-assessment Tool for Asian (OSTA) for Primary Osteoporosis in Post-menopausal Malay Women
}

\author{
DAJ Muslim, MMED Orth, EF Mohd, MMED Orth, AY Sallehudin, MMED Orth, TMS Tengku Muzaffar, \\ MMED Orth, AM Ezane*, MMED Rad \\ Department of Orthopaedics, Universiti Sains Malaysia, Kota Bharu, Malaysia \\ *Department of Radiology, Hospital Universiti Sains Malaysia, Kota Bharu, Malaysia
}

\begin{abstract}
The Osteoporosis Self-Assessment Tool for Asians (OSTA) score has been developed to identify women at risk of osteoporosis. It can be used as a screening tool for patients at risk who would benefit from bone mineral density measurement and treatment. It was developed based on data from eight countries including Malaysia. However, most subjects were of Chinese (59\%). This study evaluated the performance of OSTA among 152 post-menopausal Malay women. OSTA score calculation and DEXA scan were performed. Our results showed that the OSTA score is a good predictor of patients at risk of osteoporosis based on BMD measurements at the proximal femur. Instrument sensitivity was $87.5 \%$, specificity was $95.8 \%$, positive predictive value (PPV) was 0.538 , negative predictive value (NPV) was 0.993 , and the area under the receiver operating characteristic curve (ROC) was 0.895 . We conclude that use of the OSTA score in postmenopausal Malay women is effective and has adequate sensitivity and specificity.
\end{abstract}

Key Words:

Osteoporosis; post menopause; Malay; screening; OSTA

\section{INTRODUCTION}

Osteoporosis causes increased susceptibility to fragility fractures, and is a major cause of morbidity and mortality in postmenopausal women. Effective treatments for osteoporosis are now available. Thus, there is interest in identifying people at high risk who should receive targeted therapeutic interventions. Dual energy x-ray absorptiometry (DEXA) is the gold standard for diagnosis of osteoporosis, but it remains expensive and is not widely available. Furthermore, studies have shown that mass screening for osteoporosis using DEXA is not cost effective ${ }^{1,2}$.

The Osteoporosis Self-Assessment Tool for Asians (OSTA) score was developed by the World Health Organization
(WHO) to identify women at risk and is based simply on age and weight ${ }^{3}$. The OSTA score can be calculated by subtracting age from weight and multiplying by 0.2 . This can be written as: OSTA $=0.2[$ weight $(\mathrm{kg})-$ age (year)]. The OSTA score was developed based on data from 860 women from eight countries including Malaysia. Patients are stratified into low, intermediate and high risk groups. These three categories were arbitrarily created using the index, based on age and body weight, with cutoffs of -1 and -4 . Large differences in risk were identified using these categories. The risk of osteoporosis in the high, intermediate and low risk category was found to be $61 \%, 15 \%$ and $3 \%$ respectively. This risk index had a sensitivity of $91 \%$ and specificity of $45 \%$, with the area under the curve of 0.79 . The OSTA score was able to identify $8 \%$ of women with $61 \%$ risk of osteoporosis.

In Malaysia, a multiracial country, there is a marked difference in the incidence of hip fractures with different races. The Chinese have the highest incidence as compared to Indians and Malays. Chinese women accounted for $44.8 \%$ of all hip fractures in one local study ${ }^{4}$. We question whether the OSTA score derived from a mainly Chinese population is applicable to the Malay population.

The primary objective of this study is to determine performance of the OSTA score as a screening tool for primary osteoporosis among postmenopausal Malay women and the secondary objective is to determine whether the cutoff point of -4 appropriately identifies patients at high risk for osteoporosis. Our hypothesis is the OSTA score performs well as a screening tool for primary osteoporosis and that the cutoff point of -4 identifies patients at high risk of osteoporosis among postmenopausal Malay women. If it does not, whether any adjustments need to be made for the OSTA score cutoff to show improved sensitivity and specificity. 


\section{METHODOLOGY}

This is a prospective cross-sectional study involving 152 participants. The number of participants was determined by sample size calculation (alpha $=0.05$; power $=0.8 ; \mathrm{P} 0=0.9$; $\mathrm{P} 1=0.85 ; \mathrm{R}=0 ; \mathrm{PHI}=0.75$; required number of subjects $=138$ with additional $10 \%$ drop out). Participants were recruited from the community between 2008-2009. This study was approved by the Human Research Ethics Committee of Univesiti Sains Malaysia (USMKK/PPSP/JEPeM [(206.3.(2)]).

Dual-emission X-ray absorptiometry (DEXA) scans of the proximal femur and anteroposterior (AP) spine were performed at enrollment, using a GE Lunar Prodigy DEXA machine with ENCORE 2002 software. Malay postmenopausal women with suitable hip anatomy for evaluation of bone mineral density (BMD) were included in the study. Subjects with history of metabolic disease, presence of bone metastasis, significant renal impairment, previous bilateral oophorectomy, previous both hip fracture or prior use of any bisphosphonate were excluded from the study.

Statistical analysis was performed using Statistical Package for Social Science (SPSS) software version 12.0. Statistical significance was set at 5\%. A positive DEXA scan is the gold standard for diagnosis of osteoporosis. The sensitivity, specificity, positive and negative predictive value was calculated. Prevalence of the disease in the population sample was also calculated. The receiver operating characteristic curve (ROC) was plotted and the area under the curve was derived.

\section{RESULTS}

A total of 152 participants were included in this study. The mean age of participants was $59.4 \pm 6.4$-years-old and the mean age of menopause onset was $50.8 \pm 2.7$ years. The mean weight, height and BMI were $60.8 \pm 11.1 \mathrm{~kg}, 151.8 \pm$ $6.2 \mathrm{~cm}$ and $26.3 \pm 4.5 \mathrm{~kg} / \mathrm{m} 2$ respectively (Table I). The distribution of patients for the diagnostic test of sensitivity and specificity for proximal femur and spine are shown in Table II. For the proximal femur, the OSTA score identified 7 of 8 participants with osteoporosis and excluded 138 of 144 participants without osteoporosis, resulting in sensitivity and specificity of $87.5 \%$ and $95.8 \%$ respectively. For the spine, the OSTA score only identified 11 of 23 participants with osteoporosis and excluded 127 of 129 participants without osteoporosis, resulting in sensitivity and specificity of $47.8 \%$ and $98.4 \%$.. The prevalence of osteoporosis was $5.3 \%$ and $15.1 \%$ using proximal femur or spine DEXA results, respectively (Table III).

Cutoff points of -1 as -4 were used for diagnosis in this study $^{3}$. The results for the risk categories are summarized in
Table IV. The low risk category (index $>-1$ ) represented $69.7 \%$ of all women with only $1 \%$ of the women in this category having osteoporosis. The intermediate risk category (-1to -4$)$ represented $21.7 \%$ of participants. The high risk group (index $<-4$ ) represented only $8.6 \%$ of participants. The prevalence of osteoporosis was very high $(46.0 \%)$ in this group.

\section{DISCUSSION}

There is general agreement that postmenopausal women with a fragility fracture are at high risk for subsequent fractures, and are therefore appropriate choices for treatment to reduce the risk of future fractures ${ }^{5}$. However, only a small number of women with a history of fragility fractures are currently receiving osteoporosis treatment ${ }^{6}$. Among these women with previous fragility fractures, BMD measurement may increase their awareness of osteoporosis and lead to treatment if appropriate. Amongst women who have not yet had fractures, BMD is the best method for identification of at risk patients to consider for treatment of osteoporosis.

Even though it would be beneficial to conduct routine osteoporosis screening, it is not feasible in Malaysia due to cost constraints and insufficient availability of DEXA machines. As of 2006, there were only 44 DEXA machines available in Malaysia with the majority located in Kuala Lumpur. In 2010, it was estimated that there were 1.3 million people, aged 65-years-old of older in Malaysia ${ }^{7}$. It is therefore not feasible to screen all postmenopausal Malaysian women using DEXA.

Various risk assessment tools have been developed to identify women at risk so they can be referred for BMD measurement. Such assessment tools increase the efficiency of BMD measurement by focusing on subjects who are at increased risk. Questionnaires have been used as a tool for screening such as the Simple Calculated Osteoporosis Risk Estimation (SCORE), the Osteoporosis Risk Assessment Instrument (ORAI), the SOFSURF index and OSTA ${ }^{3,8,9}$.

Our results showed that for the T-score of proximal femur, the OSTA in postmenopausal Malay women had a sensitivity of $87.5 \%$ and specificity of $95.8 \%$. The positive predictive value (PPV) was 0.538 and negative predictive value (NPV) was 0.993. The area under the receiver operating characteristic (ROC) curve was 0.895 . This was a better result than for T-score for spine, which had a sensitivity of $47.8 \%$ and specificity of $98.4 \%$.

Sensitivity was low when DEXA measurements of the spine were used for analysis. This can be explained by the effect of vertebral fractures on the DEXA measurements. In the presence of multiple vertebral fractures, the bone density within the spine would be increased. This in turn would give 
Table I: Descriptive statistics of participating subjects

\begin{tabular}{|lcccc|}
\hline & Minimum & Maximum & Mean & Std. Deviation \\
\hline Age (year) & 48 & 82 & 59.4 & 6.4 \\
Weight $(\mathrm{kg})$ & 31.0 & 91.0 & 60.8 & 11.1 \\
Height $(\mathrm{cm})$ & 135 & 171 & 151.8 & 6.2 \\
BMI (kg/m²) & 14.2 & 41.0 & 26.3 & 4.5 \\
Menarche age (age) & 10 & 16 & 13.4 & 1.3 \\
Menopause age (age) & 45 & 58 & 50.8 & 2.7 \\
Menstruation duration (years) & 29.00 & 50.00 & 37.4 & 3.1 \\
Femur BMD (g/cm 3 ) & 0.427 & 1.299 & 0.934 & 0.164 \\
Femur T-score & -4.20 & 2.70 & 0.00 & 0.239 \\
Spine BMD & 0.461 & 2.145 & 1.055 & 1.95 \\
Spine T-score & & & & 2.7 \\
(g/cm ${ }^{3}$ OSTA score & -4.40 & 8.50 & -0.45 & 0.3 \\
\hline
\end{tabular}

Table II: Distribution of patients for determination of sensitivity and specificity of OSTA to diagnose osteoporosis compared to the gold standard DEXA scan of the proximal femur and spine

\begin{tabular}{|lccc|}
\hline & $\begin{array}{c}\text { DEXA +ve } \\
\text { \# Subjects }\end{array}$ & $\begin{array}{c}\text { DEXA -ve } \\
\text { \# Subjects }\end{array}$ & $\begin{array}{c}\text { Total } \\
\text { \# Subjects }\end{array}$ \\
\hline $\begin{array}{l}\text { Proximal Femur } \\
\text { OSTA +ve }\end{array}$ & 7 & 6 & 13 \\
OSTA -ve & 1 & 138 & 139 \\
Total & 8 & 144 & 152 \\
Spine & 11 & 2 & 13 \\
OSTA +ve & 12 & 127 & 139 \\
OSTA -ve & 23 & 129 & 152 \\
Total & & & \\
\hline
\end{tabular}

Table III: Determination of sensitivity and specificity of OSTA to diagnose osteoporosis compared to the gold standard DEXA scan of the proximal femur and spine

\begin{tabular}{|lcc|}
\hline & Proximal Femur T-score & Spine T-score \\
\hline Prevalence of osteoporosis as calculated with DEXA & 0.053 & 0.151 \\
Prevalence of osteoporosis as calculated with OSTA & 0.086 & 0.086 \\
Sensitivity (true positive fraction, TPF) & 0.875 & 0.478 \\
Specificity (true negative fraction, TNF) & 0.958 & 0.984 \\
Positive predictive value (PPV) & 0.538 & 0.846 \\
Negative predictive value (NPV) & 0.993 & 0.914 \\
Area below the ROC curve & 0.895 & 0.894 \\
\hline
\end{tabular}

Table IV: Summary of results

\begin{tabular}{|lccc|}
\hline Category & Score & Risk of osteoporosis & Per cent of women \\
\hline High risk & $<-4$ & $46.0 \%(4 / 13)$ & $8.6 \%(13 / 152)$ \\
Intermediate risk & -1 to -4 & $0 \%(0 / 33)$ & $21.7 \%(33 / 152)$ \\
Low risk & $>-1$ & $1 \%(1 / 106)$ & $69.7 \%(106 / 152)$ \\
\hline
\end{tabular}


a falsely improved DEXA measurement that does not reflect overall osteoporotic status. In the original OSTA study by Koh et $a .^{3}$, spinal BMD measurements were not included.

In the Koh study using proximal femur measurements, the sensitivity, specificity and area under the ROC curve were $91 \%, 45 \%$ and 0.79 . This is similar to our finding using the same cutoff point of -1 and -4 . Our sensitivity is slightly lower but specificity is higher. The probability of a patient with an OSTA score of less than -4 having osteoporosis, or the PPV, is $53.8 \%$. The probability of a patient with an OSTA score of more than -4 not having osteoporosis, or the NPV, is $99.3 \%$. Statistical analysis to find a better cutoff point for the Malay population was not successful in improving sensitivity and specificity. The cutoff point of -4 gave the overall best sensitivity and specificity values. These findings make the OSTA an appropriate tool for screening in postmenopausal Malay women with -4 being the best cutoff point to identify high risk of osteoporosis.

The OSTA have been compared to various clinical screening tools in several studies in different populations. Most found OSTA most useful ${ }^{8-11}$. A study by Geater et al. involving 388 postmenopausal Thai women found the OSTA to be a good predictor of osteoporosis ${ }^{12}$, with sensitivity and specificity of $93 \%$ and $61 \%$ for proximal femur and $80 \%$ and $70 \%$ for the spine, respectively. Other population based OSTA studies reported found good correlation for Thailand, Singapore, Taiwan and China study samples ${ }^{13-16}$. Similar good correlation has been reported from OSTA studies in men ${ }^{17}$.
The present study showed that OSTA is an effective tool for identifying postmenopausal Malay women at risk of osteoporosis. Using of the OSTA scoring system applying the standard cutoff point of -1 and -4, BMD can be targeted to the high risk group thereby optimising resource utilisation. With this screening tool, $87.5 \%$ of patients with osteoporosis can be diagnosed. This would mean that only $8.6 \%$ of postmenopausal women (those recognized as high risk) would need BMD examination for diagnosis of osteoporosis. Such practice would optimise the cost effectiveness of osteoporosis management.

\section{CONCLUSION}

The Osteoporosis Self-Assessment Tool for Asians (OSTA) score is superior to broad risk-factor analysis in identification of postmenopausal women with osteoporosis. It has been well studied and validated in numerous studies. The use of the OSTA in postmenopausal Malay women is effective and has high sensitivity and specificity. The standard cutoff score of -4 effectively identifies patients at high risk for osteoporosis. Using this simple tool, we are able to avoid BMD measurements in low risk women. Hence, more prudent use of limited resources can be concentrated on the use of diagnostic and therapeutic modalities for high risk women. Based on this study, the prevalence of osteoporosis was $5.3 \%$. 


\section{REFERENCES}

1. Weinstein L, Ullery B, Bourguignon C. A simple system to determine who needs osteoporosis screening. Obstet Gynecol. 1999; 93(5,1): 757-60.

2. Skedros JG, Sybrowsky CL, Stoddard GJ. The osteoporosis self-assessment screening tool: a useful tool for the orthopaedic surgeon. J Bone Joint Surg [AM] 2007; 89(4): 765-72.

3. Koh LK, Sedrine, WB, Torralba TP, Kung A, Fujiwara S, Chan SP, et al. A simple tool to identify Asian women at increased risk of osteoporosis. Osteoporos Int. 2001; 12(8): 699-705.

4. Lee CM, Sidhu JS, Pan KL Hip fracture incidence in Malaysia 1981-1989. Acta Orthop Scand. 1993; 64(2): 178-80.

5. Freedman KB, Kaplan FS, Bilker WB, Strom BL, Lowe RA Treatment of osteoporosis: are physicians missing an opportunity? J Bone Joint Surg [AM] 2000; 82(8): 1063-70.

6. Bauer DC. Osteoporotic fractures: ignorance is bliss? Am J Med. 2000 109(4): 338-9.

7. Population and Housing Census, Malaysia c2010 (2010 Census). Department of Statistics Assessed on 24th Dec 2011 Available from: Malaysia: http://www.statistics.gov.my

8. Rud B, Hilden J, Hyldstrup L, Hrobjartsson A. The Osteoporosis Self-Assessment Tool versus alternative tests for selecting postmenopausal women for bone mineral density assessment: a comparative systematic review of accuracy. Osteoporos Int. 2009; 20(4): 599-607.

9. RudB, Jensen JE, Mosekilde L, Nielsen SP, Hilden J, Abrahamsen B. Performance of four clinical screening tools to select periand early postmenopausal women for dual X-ray absorptiometry. Osteoporos Int. 2005; 16(7): 764-72.

10. Lau EM, Lee JK, Suriwongpaisal P, Saw SM, Das De S, Khir A, Sambrook P. The incidence of hip fracture in four Asian countries: the Asian Osteoporosis Study (AOS). Osteoporos Int. 2001; 12(3): 239-43.

11. Schwartz EN, Steinberg DM Prescreening tools to determine who needs DXA. Curr Osteoporos Rep. 2006; 4(4): $148-52$.

12. Geater S, Leelawattana R, Geater A. Validation of the OSTA index for discriminating between high and low probability of femoral neck and lumbar spine osteoporosis among Thai postmenopausal women. J Med Assoc Thai. 2004; 87(11): $1286-92$.

13. Chaovisitsaree S, Namwongprom SN, Morakote N, Suntornlimsiri N, Piyamongkol W. Comparison of osteoporosis self assessment tool for Asian (OSTA) and standard assessment in Menopause Clinic. Chiang Mai. J Med Assoc Thai. 2007; 90(3): $420-5$.

14. Chan SP, Teo CC, Ng SA, Goh N, Tan C, Deurenberg-Yap M. Validation of various osteoporosis risk indices in elderly Chinese females in Singapore. Osteoporos Int. 2006; 17(8): 1182-8.

15. Yang NP, Lin T, Wang CS, Chou P. Correlation of osteoporosis screening by quantitative ultrasound of calcaneus and Osteoporosis Self-Assessment Tool for Asians in Taiwanese. J Formos Med Assoc. 2004; 103(2): 130-6.

16. Lu C, Chen D, CaiY, Wei S. Concordance of OSTA and lumbar spine BMD by DXA in identifying risk of osteoporosis. J Orthop Surg Res. 2006; 1: 14.

17. Ghazi M, Mounach A, Nouijai A, Ghozlani I, Bennani L, Achemlal L, Bezza A, El Maghraoui, A. Performance of the osteoporosis risk assessment tool in Moroccan men. Clin Rheumatol. 2007; 26(12): 2037-41. 